\title{
Collaborative Networks in Support of Service-Enhanced Products
}

\author{
Luis M. Camarinha-Matos ${ }^{1}$, Hamideh Afsarmanesh ${ }^{2}$, and Bernhard Koelmel ${ }^{3}$ \\ ${ }^{1}$ Faculty of Sciences and Technology, Universidade Nova de Lisboa / Uninova, Portugal \\ cam@uninova.pt \\ ${ }^{2}$ Informatics Institute, University of Amsterdam, The Netherlands \\ h. afsarmanesh@uva.nl \\ ${ }^{3}$ CAS Software AG, Germany \\ bernhard.koelmel@cas.de
}

\begin{abstract}
The development and support of highly customized and serviceenhanced products requires new organizational structures, involving the manufacturers, customers and local suppliers in a process of co-creation. This requires the implementation of the glocal enterprise notion with value creation from global networked operations and involving global supply chain management, product-service linkage, and management of distributed manufacturing units. An approach based on cloud-computing and advanced collaboration spaces is proposed for such contexts. As application scenario the manufacturing and life cycle support of solar parks is considered.
\end{abstract}

Keywords: Collaborative networks, Service-enhanced products, Collaborative Ecosystems, Cloud computing.

\section{Introduction}

There is a growing trend in manufacturing to move towards highly customized products, ultimately one-of-a-kind, which is reflected in the term mass customization. In fact, mass customization refers to a customer co-design process of products and services which meet the needs/choices of each individual customer with regard to the variety of different product features [2], [4]. Important challenges in such manufacturing contexts can be elicited from the requirements of complex technical infrastructures, like security infrastructures, alternative energy, or illumination systems in large public buildings or urban equipments, but also in more traditional complex products such as customized kitchens:

- These products typically require a variety of competencies and resources, hardly available in a single enterprise, which calls for collaboration among several companies and individuals.

- In many cases operations are performed within a fixed solution space, characterized by stable but still flexible and responsive processes, which can highly benefit from ICT support. As a result, the costs associated with customization allow for a price level that does not imply a switch in an upper market segment. 
- A complex multi-supplier product with a high degree of customization would benefit from associated services (e.g. maintenance support, assistance wizard, etc.) leading to the notion of service-enhanced products, which are more difficult to plan and arrange than with standardized mass products.

- Customization demands that the recipients of the customized goods transfer their specific needs and desires into a concrete product specification. This calls for customers' integration into the value creation process to detailed defining, configuring, matching, and/or modifying an individual solution. Different from a do-it-yourself setting this is done in close interaction between the manufacturers and the customer, who will contribute to co-creating the product/service with the manufacturer (or provider) responsible for providing the customized solution.

From a strategic management perspective, mass customization is a differentiated strategy. Customers gain from the customization of the increment of utility and variation of a good that better fits to their needs than the best standard product attainable. The larger the heterogeneity of all customers' preferences, the larger is this gain in utility and variation. From a managerial point of view, customization can be carried out with regard to fit, style, functionality, etc. Matching the level of customization offered by a manufacturer with the customers' needs becomes a major success factor. Providing European SMEs with adequate ICT support environments for mass customization therefore gives them a leading edge over other competitors from others regions that are more competitive in mass production of standardized products.

Overcoming the economic crisis requires companies to focus on exporting, namely to act in emerging markets such as the BRIC (Brazil, Russia, India, China), which is difficult for SMEs to compete in, if working alone. In this context, many enterprises are struggling to survive in the currently turbulent markets, whilst some become leaders in gaining new markets and effectiveness by targeting their future in terms of new products and services, or some other emerging technologies, having their focus already on technical innovation and strong customer-orientation. When focusing on customizable complex products, a domain where European companies might have an opportunity, it is important to be aware of the socio-economic, traditional, cultural, and perhaps even religious context surrounding the customer. A collaborative network involving not only a network of European manufacturers, but also the customer and some local suppliers is thus beneficial and necessary. Since the main customers of complex products and technical infrastructures are public entities, it is even frequently the case that the collaboration with local suppliers is a contractual requirement.

This paper introduces the base concepts and preliminary results of the GloNet project which aims the development of the required support platforms interlinked with the development of adequate organizational and governance models to facilitate the rapid formation and effective operation of the described partnerships.

\section{A Motivating Case}

The industry-driving use case in GloNet is in the Solar Park construction sector. The norm of operation in this industry is that of one-of-a-kind production and delivery of 
products (e.g. infrastructure, photovoltaic panels, and control systems). The results (products and services) are typically delivered through complementary competences sharing between different project participants. Such operational modes naturally have implications on the way the Product Lifecycle Management (PLM) is organized between the participating organizations.

As energy costs continue to rise, businesses need smarter energy management strategies. Advances in control technology give the benefits of energy management and control systems in a single, sustainable, and flexible networked infrastructure. During a product development process, it is essential to identify the critical components, critical suppliers and also the proportion of commodities to be outsourced to each supplier, thereby deciding on the structure of a virtual enterprise of involved companies.

The Infranet Partners is a network of SMEs specializing in the control technology solutions based on production and provision of advanced control technologies. This network was established in 1999 with 4 founding members and today there are some 20 partners in this network. In photovoltaic solutions around the world, products from the Infranet Partners are used to monitor and control solar park units and their related processes.

Solar parks include a number of subsystems - security, lighting, elevators, power, safety, and HVAC (Heating, Ventilating, and Air Conditioning) — that are crucial to a well-run system. But most of them operate separately or, at best, are connected through a series of costly, hard-to-maintain gateways to a single humanmachine interface. The Infranet Partners invested on the concept of open control systems, where manufacturers follow independently maintained interoperability guidelines that create a single, unified automation system. Embedded technology and infrastructure products are at the heart of these open systems.

Aiming at a new generation of systems, integrating remote support services, and in order to better serve customers, the Infranet Partners aim to improve their current operation as a collaborative network, through:

- Creating a comprehensive pool of Technology and Application resources.

- Serving the customers as a single organization, offering locally adapted solutions from this shared pool.

- Answer the demand for holistic user-centered solutions (customers demand „,complete "e solutions out of one hand).

- Focusing on core competencies of each partner in the virtual enterprise: delegating the process of value generation into the supply chain.

- Combining their products range under the Infranet Partners brand and providing services associated to the products along their life cycle.

- Providing a comprehensive product range and support backed by frequent cross training.

- Sharing technical support and knowledge of different markets to provide solutions for customers.

- Sharing marketing information using an advanced dynamic groupware marketing tool to enable them to act faster to meet customer requirements.

- Reaching a smooth collaboration environment involving customers and local suppliers in their target market locations (e.g. India). 
Example services associated to the product life cycle include:

- Remote monitoring and diagnosis

- Remote supervision

- Predictive maintenance

- Product usage: training, assistance, interaction.

Market drivers in complex industrial infrastructures like solar plants come with increased usage of controlling software and electronics as a distinguishing feature, coupled with changes in manufacturing such as globalization, increased competition, higher safety needs and faster serviceability. This has led to challenging new requirements for content-rich and price-competitive products. In this domain, the latest product developments incorporate new intelligent features with increasing complexity and the use of services, electronics and software as differentiators in the marketplace. These features require added manufacturing know-how along the value chain to drive the next level of operational efficiency and performance. The development of these complex interlaced systems over the entire product lifecycle represents an increasing challenge for all manufacturers and their suppliers.

As such, the following are some challenges faced by the manufacturers within the planned GloNet Virtual Enterprise:

- Mechanical, construction, electrical, and software product development have traditionally grown up as separate silos of expertise and technology that need to be brought together early in the development lifecycle of the product. Furthermore clients of such one-of-a-kind production industry demand user-centred customised products and services that a single organisation may not be able to deliver. GloNet is developing a platform, tools and governance principles to support effective creation and operation of the virtual enterprise and its interaction with the customer.

- There is no single management system for capturing product data at network level. Mechanical, electrical, and software data are often handled in separate document management systems with no automated sharing of data between the systems or links between data. With GloNet, all relevant documents can be stored centrally and called upon via web-service APIs, providing a single source of all productrelated information along its life cycle.

- There is no commercial system design environment currently available for this application field. At the moment home-grown systems are patched together, which are expensive to develop and maintain. GloNet allows for a suitable extension.

- Due to the growing number of product variants and increasingly short lifecycles, costs for developing physical prototypes have become increasingly prohibitive, therefore the knowledge of the engineers have to distributed within projects, but limited to one project. GloNet provides role-based and differentiated authentication mechanisms to support this requirement. Furthermore, all requests are tracked.

- The Infranet partners are realizing embedded software in an area of competitive differentiation and are investigating how to improve and streamline their software processes along the value chain. There are no commercially available systems for keeping the software version synchronized with the mechanical and electrical product data. GloNet allows for a suitable extension. 
- Due to the increased electronic content and provision of value added services like services associated to the product (solar park) along its life cycle (i.e. service design, decentralised support, proactive maintenance). Managing the supply chain without the basic Virtual Enterprise and tying to deal with as-shipped product structure is a growing challenge. GloNet provides a collaborative solution.

Within GloNet we envision a best practice in Virtual Enterprise management for complex industrial units like solar plants that will address these challenges.

\section{The Conceptual Approach}

GloNet adopts a cloud-based approach [5] for the development of such support environment so that its supporting services can dynamically upgrade without influencing the nodes and stakeholders in the environment. As such the environment stakeholders procure the use of upgraded services, which are always available through the cloud, rather than buying static products which typically need to be installed and maintained by the stakeholders on adequately supporting computing facilities. Specifically, over the cloud two virtual spaces are considered (Fig. 1):

- Collaborative solution space - where manufacturers, local suppliers and customers meet to co-design the product (and associated services).

- Service provision space - a "registry" of the products, along their life-cycle, where the customer can have access to the specific services associated to the customized product.

Fig. 2 illustrates the key concepts involved in GloNet and main relationships among these concepts.

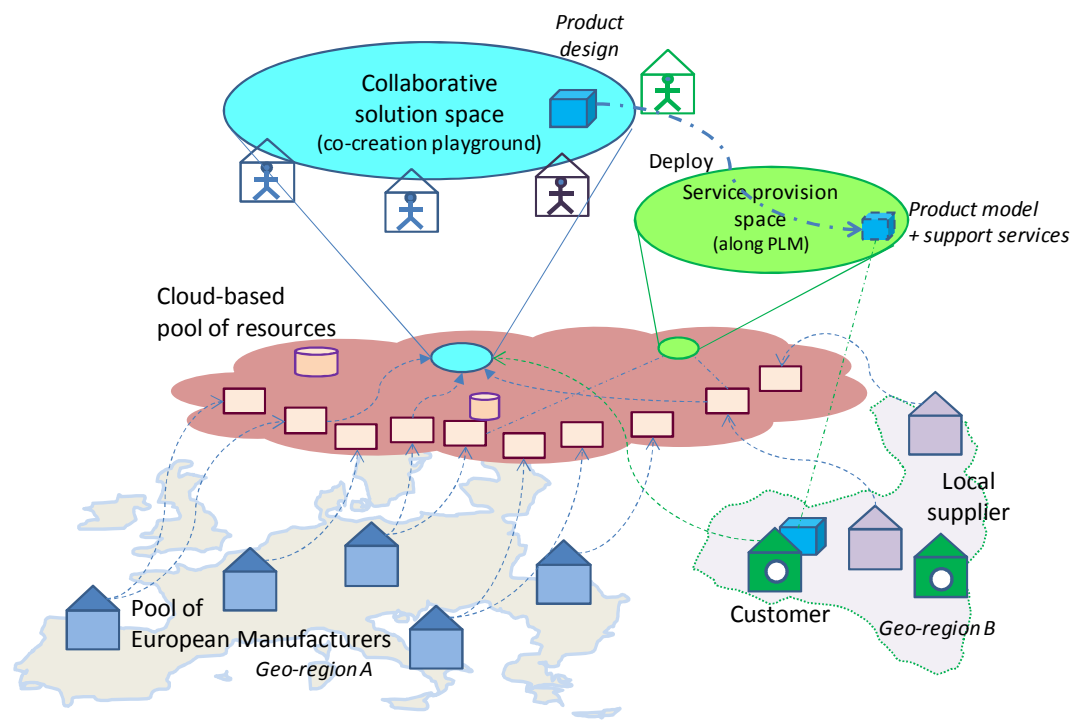

Fig. 1. Virtual spaces in GloNet 
Organizational structures. Taking into account the possibilities offered by emerging technologies for collaboration support, including adaptive business process and business services modelling, participative environments, and social software, GloNet envisions a dynamic business ecosystem where interconnections between players and their roles are dynamically adjusted to the business opportunities. Therefore the project considers:

- Inter-play of long-term and short-term networks, i.e. Virtual enterprise Breeding Environments (VBE) and dynamic goal-oriented virtual enterprises (VE) [1].

- Interplay of manufacturers' network with customers and local suppliers (implementing the glocal enterprise and co-creation notions).

- Development phase network and PLM support network, in other words, a virtual enterprise might be organized for the product development and dissolve after the product is delivered, while another virtual enterprise, eventually sharing some members with the first one, might be organized to support the product along its life cycle.

In order to properly design the organizational structures, GloNet is starting with matching needs (analysis of current practices) with state of the art and emerging organizational models as identified in the disciplines of collaborative networks and organizational ecology and also briefly pointed out in the FInES roadmap [6].

Particularly in what concerns the involvement of the customers (and local suppliers) in an open innovation process, GloNet goes beyond the traditional process in which customer inputs are collected at the end of the process. Through the proposed collaboration space, GloNet exploits the synergies created by the involvement of the customer and local suppliers in all phases of the product development and life cycle support.

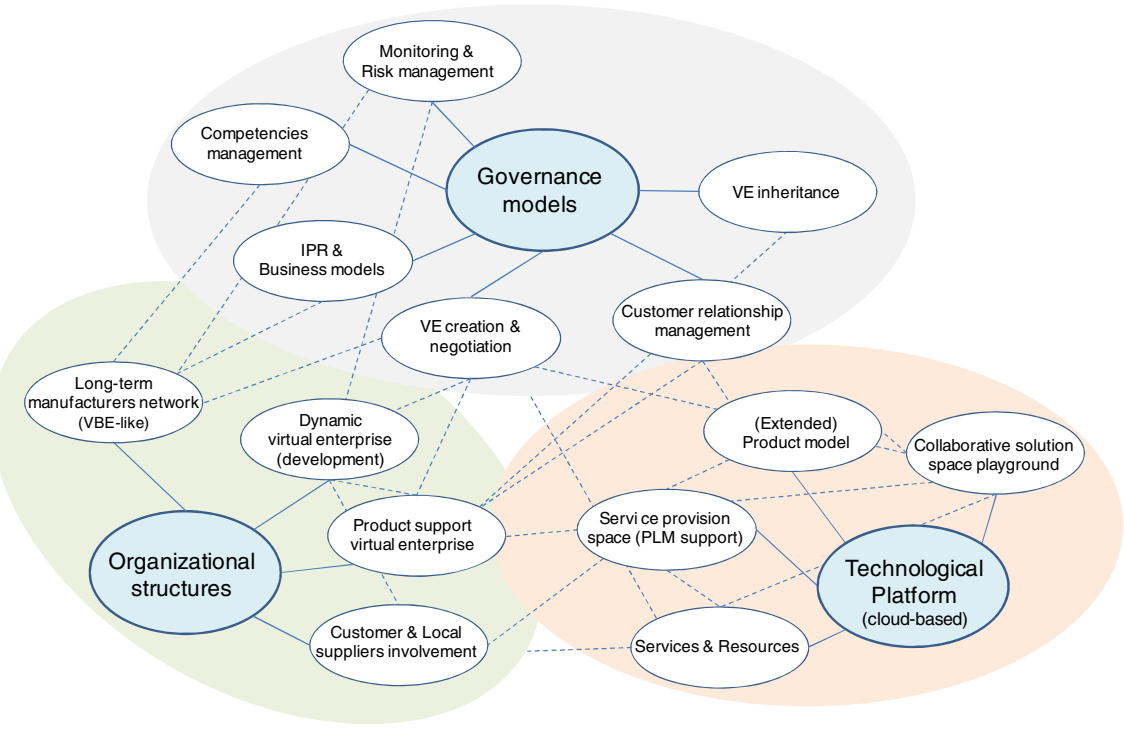

Fig. 2. Main supporting concepts 
Technological platform. Regarding the base platform the following main characteristics are planned:

- Cloud-based infrastructure, based on open-source technologies, adopting relevant standards and based on OSGi.

- Incremental pool of services, knowledge, and other resources (scalability characteristic).

- Supporting the notion of extended product - combination of physical product with a set of linked support services (e.g. maintenance, remote diagnosis, remote user assistance, training, ...). A product model will become available in the Service Provision Space as a single entry point for product-related information and services along its life cycle. The product support virtual enterprise will naturally be linked to this product model.

- Besides the cloud-based platform, the environment includes two main (virtual) spaces: (i) Collaborative solution space - where new products are developed / customized (co-creation/mass customization) through the interplay of the various stakeholders (product development virtual enterprise); (ii) Service provision space - where models of products and associated services are kept along the product life cycle, supported by the product support virtual enterprise.

Governance models. Under this pillar the following main concepts and support functionalities are developed:

- Competencies management and competencies gap analysis (business intelligence).

- IPR \& business models for co-created products.

- VE creation \& negotiation principles, including readiness assessment and value systems alignment analysis.

- Performance monitoring and risk assessment, including aspects of "collaboration emotional health".

- Customer relationship management ("who owns the customer", brokerage services, etc.).

- VE inheritance - transition from product development VE to PLM support VE.

The chosen real industry pilot scenario, on the other hand, serves not only the demonstration and validation purposes, but also helps focusing on the requirements elicitation and system design and development.

\section{Cloud Based Platform}

GloNet base platform is based on the Cloud Development Stack model CAS OPEN [3], which is a standardized objective framework for cloud-offerings. CAS OPEN has a set of broad-based and deep capabilities that enable cost-effective development in the Cloud. The main objective that is addressed by the base GloNet architecture is the provision of a platform that allows for extensive customization and extensibility of SaaS for virtual enterprise functionalities offerings without compromising reliability and security. The architecture is intended not only to guide the software development 
but also to provide a technology-independent model of the platform. A base framework is assumed, providing the basis for interoperability, incremental service addition, security, and privacy mechanisms.

In order to increase the interoperability and consistency of engineering and manufacturing solutions with efficient collaboration approaches, the GloNet System will be built on top of an open service platform compliant with the OSGi framework.

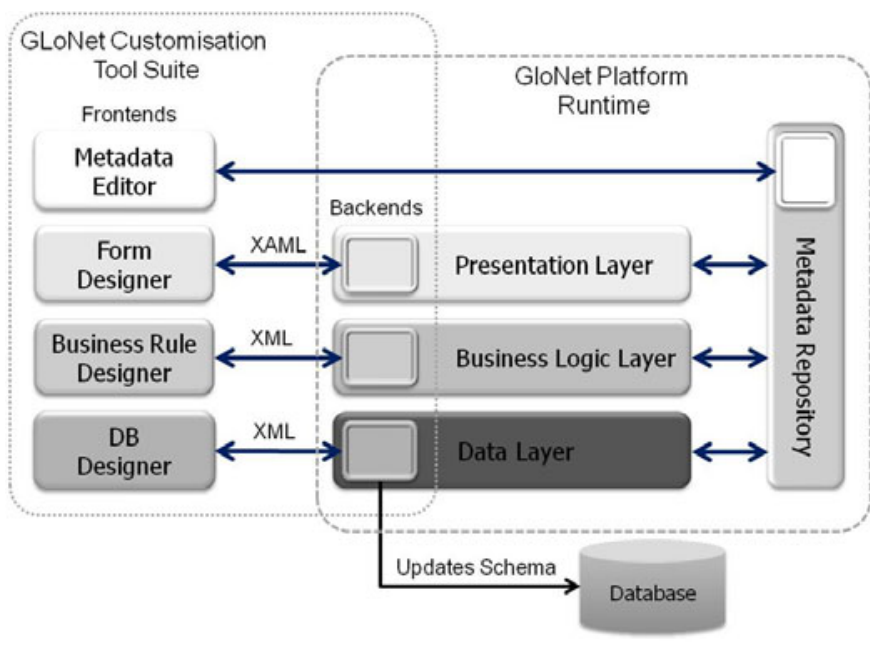

Fig. 3. GloNet base platform architecture

The system will consist of two major components: The Glo Net-Server, conceived as a central open cloud service infrastructure, consisting of basic functions and components, which are responsible for the business logic, the virtualization of "on premise solutions" as well as for interlinking and aggregating information sources of software services running on the platform. As a consequence of the SaaS customization scenario, development tools consist of two parts: (i) a backend, which is an integral part of the corresponding layer (data, logic, or presentation) and is available not only during the development of a solution, but also during runtime of a GloNet platform instance, (ii) a designer (design-time tool), which is used to create the configuration specifications that are accepted by the corresponding backend. Because a layer's behavior must be adaptable during runtime of a GloNet platform instance, the truly challenging part of the customization mechanism is actually the backend. Since almost all concepts and logic that are needed to support a SaaS customization are part of the backend, the latter will be the focus of the customization tools being developed in the course of the GloNet project.

The ability to use Web services enables the developers of solutions and customizations to leverage already existing (and tested) solutions for particular highly-specialized tasks within their apps, instead of having to reinvent the wheel. For example, an app or app extension for collaborative contact management could invoke an external service to perform a check if a pre-product is available within the value chain. This will be also the approach for integration with legacy systems found within some tenant's organization. An example would be to use a Web service to integrate a tenant's legacy ERP system. Naturally external services are expected to come with a variety of programming interface design conventions (atomic invocations, complex protocol-based conversations, etc), and different implementation technologies (SOAP-based and REST-based). For reasons of completeness and flexibility, the GloNet platform must support the integration of apps with any type of external 
service. On the other hand, for reasons of facilitating configuration-based integration of external services as an alternative (but not replacement) to programming-based integration, which should still be possible to do, the GloNet platform also needs to set some constraints on service design conventions and implementation technologies.

The ubiquity of the cloud helps overcome the previous need to house all the members of the team under one roof. The cloud places communications and coordination within the affordable reach of every SME.

\section{Advanced Functionalities}

On top of the cloud-based platform a set of advanced functionalities are designed:

Customized service-enhanced product specification. GloNet addresses ordering complex multi-supplier products which require high degree of customization, and tailoring to varied set of customer's criteria. One of the aims is thus to facilitate detailed specification of such complex customized products, when also enhanced with their required business services, e.g. to support their maintenance, insurance, etc. To achieve this, the development of the following supporting tools and intelligent userbased interfaces is going on:

- Services for customers/local suppliers to iteratively specify product details starting with the semi-open data platform of all sub-product alternatives, the system will facilitate the iterative process of achieving the exact specific final selection of required sub-products, agreed by the customer to be ordered.

- Dynamically customizable set of business services enhancing the product based on the customer profile established dynamically in the previous services, here we focus on the development of a recommendation tree for offering to customer all alternatives of enhancing services around the ordered product. An adaptive dialogue structure is also designed on top of the recommendation tree that uses service reputation for promoting the services to the customer.

Consortia formation and operation support. These functionalities are intended to support the networked multi-stakeholder organizational structures involved in the service-enhanced product development and support. Thus they include the elaboration of models, development of support tools (services) and governance principles regarding: (i) Long-term base networks (manufacturers), (ii) Dynamic goal-oriented virtual enterprises (also involving the customer and local suppliers), (iii) Virtual enterprises for product life cycle support. Furthermore, process monitoring, risk forecasting, and management are also included. Thus the main services here include:

- Services for management of long-term base network - to support the management of long-term alliances of manufacturers, which typically assume the form of a VBE (Virtual organizations breeding environment) with flexible boundaries (different levels of membership). Support services include partners' profiling and competency management, competencies gap analysis, collaboration readiness assessment, value systems alignment assessment, trust management, incentives and assets management.

- Services for dynamic consortia formation and order work plan - a collection of 
software services for dynamic consortia formation and monitoring involvement of customers and local suppliers.

- Services for consortium risk forecasting and management - concerned with identification and characterization of risks in a collaborative context as well as their forecasting, monitoring and launching of preventive / corrective actions. The relationships between risks and competencies and expectations are analyzed. Since emotional / affective aspects play a critical role in the levels of commitment and performance of network members, attention is also devoted to monitoring and promotion of the collaboration "health" at network level.

\section{Conclusions and Further Work}

In support of complex service-enhanced products along their life cycle, the approach followed in GloNet aims to: (i) develop a novel way to commonly represent/provide information and knowledge which needs to be shared/exchanged among different stakeholders in the collaborative environment as dynamic software services that may upgrade in time; (ii) generate user-customized interfaces which dynamically adjust to different stakeholders, supporting their access and visualization needs; (iii) provide these services through the cloud, to be available to anybody, at any time, from anywhere, (iv) demonstrate how a broker in very close contact with the customer who gives an order, can iteratively retrieve all needed information to step by step design the customer order and finally presenting the solution that is accepted by the customer, (v) support the negotiation among all involved parties, (vi) generate a workflow from the accepted/negotiated solution, which will then be automatically monitored, while also available for monitoring by the involved stakeholders, during its execution, (vii) the automatic monitoring aims will forecast potential risks, and will suggest prevention measures to the broker during the execution of the order.

A preliminary system and exemplary services have been designed based on the needs identified in the context of solar plants. Next phases involve the field validation of the approach and tools as well as the assessment of their potential applicability to other contexts.

Acknowledgments. This work was funded in part by the European Commission through the GloNet project.

\section{References}

1. Camarinha-Matos, L.M., Afsarmanesh, H.: Collaborative Networks: Reference Modeling. Springer, Heidelberg (2008)

2. Cox, H., Mowatt, S.: Consumer-driven innovation networks and e-business management systems. Qualitative Market Research: An International Journal 7(1), 9-19 (2004)

3. Koelmel, B.: Enable secure access - CAS Open - SaaS Framework. Heidelberg Innovation Forum (2008)

4. Ogawa, S., Piller, F.T.: Collective Customer Commitment: Reducing the risks of new product development. MIT Sloan Management Review 47 (2006)

5. Ommeren, E., Duivestein, S., de Vadoss, J., Reijnen, C., Gunvaldson, E.: Collaboration in the Cloud. Microsoft and Sogeti (2009) ISBN 9789075414240

6. FInES, Research Roadmap. European Commission, FInES Cluster (June 2010) 